\title{
Corrigendum: Phosphoregulation of Ire1 RNase splicing activity
}

Filippo Prischi, Piotr R. Nowak, Marta Carrara \& Maruf M.U. Ali

Nature Communications 5:3554 doi: 10.1038/ncomms4554 (2014); Published 7 Apr 2014: Updated 17 Dec 2014

In the Methods section of this Article, an incorrect sequence was provided for the single-strand RNA probe used for in vitro RNA splicing assays. The correct sequence is 5'-Cy3-GAGUCCGCAGCACUC-BHQ2-3'. 